\title{
A multi objective geometric programming approach for electronic product pricing problem
}

\author{
Mohsen Fathollah Bayati ${ }^{\mathbf{a}}$ and Ahmad Makui ${ }^{\mathbf{a}^{*}}$
}

${ }^{a}$ Department of Industrial Engineering, Iran University of Science \& Technology, Tehran, Iran

\begin{tabular}{l}
\hline A R T I C L E I N F O \\
\hline Article history: \\
Received October 122010 \\
Received in revised form \\
3 February 2011 \\
Accepted 3 February 2011 \\
Available online \\
4 February 2011 \\
\hline Keywords: \\
Optimal pricing \\
Optimization \\
Multi-objective decision making \\
Geometric programming \\
Compromise programming
\end{tabular}
A B S T R A C T

Nowadays electronic commerce plays an important role in many business activities, operations, and transaction processing. The recent advances on e-businesses have created tremendous opportunities to increase profitability. This paper presents a multi-objective marketing planning model which simultaneously determines efficient marketing expenditure, service cost and product's selling price in two competitive markets. To solve the proposed model, we discuss a multi-objective geometric programming (GP) approach based on compromise programming method. Since our proposed model is a signomial GP and global optimality is not guaranteed for the problem, we transform the model to posynomial form. Finally, the solution procedure is illustrated via a numerical example and a sensitivity analysis is presented.

(C) 2011 Growing Science Ltd. All rights reserved.

\section{Introduction}

Making an appropriate pricing and marketing strategy is a crucial management issue in E-commerce. The most fundamental rule of e-commerce is to have internet infrastructures such as email, websites, etc to build a bridge among customers, partners and suppliers (Lee et al., 2006). Today, the digital-good providers normally use internet to perform e-commerce transactions. They also try to implement many strategies based on the consumer's preference to gain more profit creating a competitive advantage against their competitors (Lee et al., 2006; Bhargava et al., 2001). Electronic products are all types of products sold on the internet based infrastructure. Therefore, pure digital and also physical products are typical examples of electronic products (Fathian et al., 2009). Optimal pricing marketing strategy plays an important role on electronic businesses (Chen et al., 2006; Chun \& Kim, 2005). Some researchers consider the effects of pricing and marketing expenditure on products. For example, Demand of many products is normally considered as a function of price, marketing, research and development, etc. Sadjadi et al. (2005) and Serel (2009) studied the joint production planning using geometric * Corresponding author. Tel: +9821-73225004 
programming. Fathian et al. (2009) studied the effects of pricing and marketing expenditure on electronic products. Furthermore, some researches assumed that the price or cost can also be considered as independent decision variable (Sadjadi et al., 2005; Elmaghraby \& Keskinocak, 2003; Jornsten \& Uboe, 2009).

In this paper, pricing and marketing strategies are determined in two competitive markets. In the proposed model, the primary objective is to maximize profit in the first market. Then the objective of the second market is to remain optimal while keeping the optimality of the first objective. Geometric Programming (GP) method is used to find the optimal solution of the proposed model. GP is a mathematical programming technique which has been widely used in engineering design research (Beightler \& Philip; Duffin et al., 1967; Sadjadi et al., 2005; Jung \& Klein, 2001; Abad, 1988; Kim \& Klein, 1998; Lee, 1993; Lee et al., 1996) to determine the optimal price and lot size. We extend the previous optimal pricing of Fathian et al. (2009) where producer faces with two competitive markets. They investigated a pricing model for electronic goods, where the resulted model considers demand as a function of price, marketing expenditure and service business in two markets. The proposed model of this paper differs from the previous works where we consider optimal pricing for two distinctive markets. Furthermore, unlike most of the earlier researches the proposed model determines the optimal value of service cost in additional to optimal price and marketing expenditure. Finally, we apply compromise programming to solve the proposed model.

This paper is organized as follows: in the next section, we represent problem statement, notations and assumptions. Furthermore our proposed model is represented in section 2 . The mathematical analysis and solution procedure is discussed in section 3. The implementation of the proposed method is illustrated via a numerical example and sensitivity analysis are given in section 4. Finally, in section 5, some conclusions are drawn from the discussion.

\section{Problem statement}

Consider an optimal pricing, marketing and service strategy for a single electronic product in two competitive markets where demand is affected by selling price, marketing expenditure and service cost and production cost depends on demand. The basic objective of the present study is to maximize the total profit in two markets. The following summarizes the necessary notation and assumption for the proposed model.

\subsection{Notations}

For $i=1,2$ consider the following notations:

$D_{i} \quad$ Demand per unit time

$C_{i} \quad$ Production cost per unit

$M$ Marketing expenditure (decision variable)

$S$ Service expenditure (decision variable)

$P_{i} \quad$ Unit selling price (decision variable)

$k_{i} \quad$ Scaling constant for demand $\alpha_{i} \quad$ Price elasticity to demand

$\beta_{i} \quad$ Lot size elasticity to production unit cost

$\gamma_{i} \quad$ Marketing expenditure elasticity to demand

$\delta_{i} \quad$ Service cost elasticity to demand

$r_{i} \quad$ Scaling constant for unit production cost

$\pi_{i} \quad$ Manufacturer's revenue

\subsection{Assumptions}

The following assumption holds for the proposed model of this paper. 
1. Demand is a function of price, marketing expenditure and service expenditure in two markets i.e.,

$$
D_{i}=k_{i} P_{i}^{-\alpha_{i}} M^{\gamma_{i}} S^{\delta_{i}} \quad \alpha_{i}>1,0<\gamma_{i}<1,0<\delta_{i}<1, i=1,2 .
$$

The scaling constant $k_{i}$ represent other related factors and the assumption $\alpha_{i}>1$ confirms that demand increases as price is reduced. Note that parameters of Eq. (1) can be easily estimated by using linear regression to the logarithm of the function (Sadjadi et al., 2005).

2. The production unit cost is defined as a power function of demand and $r_{i}$ is the scaling constant for unit production cost.

$$
C_{i}=r_{i} D_{i}^{-\beta_{i}} \quad 0<\beta_{i}<1 .
$$

The exponent $\beta_{i}$ represents demand elasticity of unit production cost with $0<\beta_{i}<1$. This function is similar to the function considered by Lee (1993) and Fathian et al., (2009).

\subsection{The proposed model}

The proposed model of this paper determines the price, marketing expenditure and service cost in order to maximize the profit in two competitive markets. For each market, we have following objective function,

$\max \Pi(P, M, S)=$ Total revenue- Production cost- Marketing cost- Service cost

Hence, we have below two objective functions:

$$
\max \Pi_{1}\left(P_{1}, M, S\right)=P_{1} D_{1}-C_{1} D_{1}-M D_{1}-S D_{1}, \quad \max \Pi_{2}\left(P_{2}, M, S\right)=P_{2} D_{2}-C_{2} D_{2}-M D_{2}-S D_{2}
$$

Substituting Eqs. (1) and (2) in Eq. (4) and simplifying the total profit per unit time in two markets yields,

$$
\begin{aligned}
& \max \Pi_{1}\left(P_{1}, M, S\right)=k_{1} P_{1}^{1-\alpha_{1}} M^{\gamma_{1}} S^{\delta_{1}}-r_{1} k_{1}^{1-\beta_{1}} P_{1}^{\alpha_{1} \beta_{1}-\alpha_{1}} M^{\gamma_{1}-\beta_{1} \gamma_{1}} S^{\delta_{1}-\beta_{1} \delta_{1}}-k_{1} P_{1}^{-\alpha_{1}} M^{\gamma_{1}+1} S^{\delta_{1}}-k_{1} P_{1}^{-\alpha_{1}} M^{\gamma_{1}} S^{\delta_{1}+1} \\
& \max \Pi_{2}\left(P_{2}, M, S\right)=k_{2} P_{2}^{1-\alpha_{2}} M^{\gamma_{2}} S^{\delta_{2}}-r_{2} k_{2}^{1-\beta_{2}} P_{2}^{\alpha_{2} \beta_{2}-\alpha_{2}} M^{\gamma_{2}-\beta_{2} \gamma_{2}} S^{\delta_{2}-\beta_{2} \delta_{2}}-k_{2} P_{2}^{-\alpha_{2}} M^{\gamma_{2}+1} S^{\delta_{2}}-k_{2} P_{2}^{-\alpha_{2}} M^{\gamma_{2}} S^{\delta_{2}+1} .
\end{aligned}
$$

Note that both objective functions are signomial GP problems. As the global optimality is not guaranteed for a signomial problem (Duffin et al., 1967), the above problem is modified into the posynomial GP problem with one additional variable and constraint. This technique was developed by Duffin et al. (1967). It is assumed that there are lower bounds $Z_{1}$ and $Z_{2}$ for the objective functions such that maximization of $Z_{1}$ and $Z_{2}$ (or minimizing $Z_{1}^{-1}$ and $Z_{2}^{-1}$ ) is equivalent to maximize the objective values. Therefore, the above problems are modified as follow,

$$
\begin{array}{ll}
\min & Z_{1}^{-1} \\
\text { subject to } & k_{1} P_{1}^{1-\alpha_{1}} M^{\gamma_{1}} S^{\delta_{1}}-r_{1} k_{1}^{1-\beta_{1}} P_{1}^{\alpha_{1} \beta_{1}-\alpha_{1}} M^{\gamma_{1}-\beta_{1} \gamma_{1}} S^{\delta_{1}-\beta_{1} \delta_{1}}-k_{1} P_{1}^{-\alpha_{1}} M^{\gamma_{1}+1} S^{\delta_{1}}-k_{1} P_{1}^{-\alpha_{1}} M^{\gamma_{1}} S^{\delta_{1}+1} \geq Z_{1}, \\
& P_{1}, M, S, Z_{1}>0 .
\end{array}
$$

$\min Z_{2}^{-1}$

subject to $\quad k_{2} P_{2}^{1-\alpha_{2}} M^{\gamma_{2}} S^{\delta_{2}}-r_{2} k_{2}^{1-\beta_{2}} P_{2}^{\alpha_{2} \beta_{2}-\alpha_{2}} M^{\gamma_{2}-\beta_{2} \gamma_{2}} S^{\delta_{2}-\beta_{2} \delta_{2}}-k_{2} P_{2}^{-\alpha_{2}} M^{\gamma_{2}+1} S^{\delta_{2}}-k_{2} P_{2}^{-\alpha_{2}} M^{\gamma_{2}} S^{\delta_{2}+1} \geq Z_{2}$, $P_{2}, M, S, Z_{2}>0$. 
Since, $Z_{1}, Z_{2}>0$, the above constraints can be rearranged. Hence, problems (6) and (7) can be transformed into the following forms,

$$
\begin{array}{ll}
\min Z_{1}^{-1} & \\
\text { subject to } & k_{1}^{-1} P_{1}^{\alpha_{1}-1} M^{-\gamma_{1}} S^{-\delta_{1}} Z_{1}+r_{1} k_{1}^{-\beta_{1}} P_{1}^{\alpha_{1} \beta_{1}-1} M^{-\beta_{1} \gamma_{1}} S^{-\beta_{1} \delta_{1}}+P_{1}^{-1} M+P_{1}^{-1} S \leq 1, \\
& \\
& P_{1}, M, S, Z_{1}>0 . \\
\min \quad Z_{2}^{-1} & \\
\text { subject to } & k_{2}^{-1} P_{2}^{\alpha_{2}-1} M^{-\gamma_{2}} S^{-\delta_{2}} Z_{2}+r_{2} k_{2}^{-\beta_{2}} P_{2}^{\alpha_{2} \beta_{2}-1} M^{-\beta_{2} \gamma_{2}} S^{-\beta_{2} \delta_{2}}+P_{2}^{-1} M+P_{2}^{-1} S \leq 1, \\
& P_{2}, M, S, Z_{2}>0 .
\end{array}
$$

Models (8) and (9) are primal posynomial geometric programming problems.

\section{Mathematical analysis}

\subsection{Multi objective optimization problem}

A multi objective optimization problem (MOP) is defined to determine a vector of decision variables within a feasible region to minimize or maximize a vector of objective functions that usually conflict with each other. Such a problem can be formulated as follow,

$\max \left\{f_{1}(X), f_{2}(X), \ldots, f_{m}(X)\right\}$

subject to $g(X) \leq 0$,

where $X$ is vector of decision variables; $f_{i}(X)$ is the $i$ th objective function; and $g(X)$ is constraint vector. A decision vector $X$ dominates a decision vector $Y$ (also written as $\mathrm{X} \succ \mathrm{Y}$ ) if:

$f_{i}(X) \leq f_{i}(Y)$ for all $i \in\{1,2, \ldots, m\}$

and

$f_{i}(X)<f_{i}(Y)$ for at least one $i \in\{1,2, \ldots, m\}$

Pareto optimal vectors are decision vectors that are not dominated by any other decision vector. In these solutions no objective can be improved without getting worse from, at least, another objective. There are various solution methods to solve the MOP. Some of the most widely used techniques are: sequential optimization, weighting method, goal programming, goal attainment, distance based method and direction based method. For a comprehensive study of these approaches, see Szidarovsky et al. (1986). The set of multi-objective optimization problem is convex if all the objective functions and the feasible region are convex.

\subsection{Compromise programming method}

In this method, the distance between some reference point and the feasible objective region is minimized. The decision maker has to select the reference point and the matrix for measuring the distances. In this way, the multiple objective functions are transferred into a single objective function. We suppose that the weighting coefficient $w_{r}$ are real numbers such that $w_{r} \geq 0 \forall r=1,2, \ldots, k$ and $\sum_{r=1}^{k} w_{r}=1$. The weighted $\mathrm{L}_{\mathrm{q}}$-problem for minimizing distance is stated as: 
$\min \quad L_{q}(f(x))=\left(\sum_{r=1}^{k} w_{r}\left|f_{r}(x)-f_{r}\left(x^{*}\right)\right|^{q}\right)^{\frac{1}{q}}$

subject to $x \in X \quad$ for $1 \leq q \leq \infty$,

where $\mathrm{X}$ is a set of constraints, such that $X=\left\{x \in R^{n} \mid g_{i}(x) \leq b_{j}, j=1,2, \ldots, m\right\}$.

\subsection{Compromise programming method to solve the proposed model}

The multi objective marketing problem may be solved by several techniques including hybrid method and compromise programming method which is implemented for the proposed model of this paper. In this method the objective functions are combined to a single objective function. Let $w_{r} \geq 0, r=1,2$ are the normalized weights (i.e. $w_{1}+w_{2}=1$ ) corresponding to the objective functions $Z_{1}$ and $Z_{2} \cdot \Pi_{1}$ and $\Pi_{2}$ are the ideal objective values of $Z_{1}$ and $Z_{2}$, respectively. Ideal objective values can be obtained by using GP method (see appendix A). According to Miettinen (1999) the weighted $\mathrm{L}_{\mathrm{q}}$-problem is as follows,

$\min U_{q}\left(P_{1}, P_{2}, M, S, Z_{1}, Z_{2}\right)=\left(w_{1}\left(Z_{1}^{-1}-\Pi_{1}^{-1}\right)^{q}+w_{2}\left(Z_{2}^{-1}-\Pi_{2}^{-1}\right)^{q}\right)^{\frac{1}{q}}$

For $1 \leq q \leq \infty$.

Case 1. For $q=1$ the problem (10) is given as:

$\min U_{1}\left(P_{1}, P_{2}, M, S, Z_{1}, Z_{2}\right)=w_{1}\left(Z_{1}^{-1}-\Pi_{1}^{-1}\right)+w_{2}\left(Z_{2}^{-1}-\Pi_{2}^{-1}\right)$

Since $w_{1}, w_{2}, \Pi_{1}$ and $\Pi_{2}$ are independent parameters, we can rearrange the problem (11) as follow,

$\min U_{1}\left(P_{1}, P_{2}, M, S, Z_{1}, Z_{2}\right)=\left[w_{1} Z_{1}^{-1}+w_{2} Z_{2}^{-1}\right]-\left(w_{1} \Pi_{1}^{-1}+w_{2} \Pi_{2}^{-1}\right)$

Hence, it is enough to solve the following problem,

$\min V_{1}\left(Z_{1}, Z_{2}\right)=w_{1} Z_{1}^{-1}+w_{2} Z_{2}^{-1}$

subject to $k_{1} P_{1}^{1-\alpha_{1}} M^{-\gamma_{1}} S^{-\delta_{1}} Z_{1}+r_{1} k_{1}^{-\beta_{1}} P_{1}^{\alpha_{1} \beta_{1}-1} M^{-\beta_{1} \gamma_{1}} S^{-\beta_{1} \delta_{1}}+P_{1}^{-1} M+P_{1}^{-1} S \leq 1$,

$k_{2} P_{2}^{1-\alpha_{2}} M^{-\gamma_{2}} S^{-\delta_{2}} Z_{2}+r_{2} k_{2}^{-\beta_{2}} P_{2}^{\alpha_{2} \beta_{2}-1} M^{-\beta_{2} \gamma_{2}} S^{-\beta_{2} \delta_{2}}+P_{2}^{-1} M+P_{2}^{-1} S \leq 1$,

$P_{1}, P_{2}, M, S, Z_{1}, Z_{2}>0$.

where $U_{1}\left(P_{1}, P_{2}, M, S, Z_{1}, Z_{2}\right)=\left(V_{2}\left(Z_{1}, Z_{2}\right)-w_{1} \Pi_{1}^{-1}-w_{2} \Pi_{2}^{-1}\right)$. Model (17) is a posynomial GP and can be solved globally by its dual problem (Duffin et al., 1967).

Case 2. For $q=2$ the problem (14) is given as:

$\min U_{2}\left(P_{1}, P_{2}, M, S, Z_{1}, Z_{2}\right)=\left[w_{1}\left(Z_{1}^{-1}-\Pi_{1}^{-1}\right)^{2}+w_{2}\left(Z_{2}^{-1}-\Pi_{2}^{-1}\right)^{2}\right]^{\frac{1}{2}}$

Problem (18) cannot be transformed into posynomial form. Hence, in order to minimize this problem, we consider the following objective function that is near to (18). 
$\min$

$$
V_{2}\left(Z_{1}, Z_{2}\right)=w_{1} Z_{1}^{-2}+w_{2} Z_{2}^{-2}
$$

subject to $k_{1} P_{1}^{1-\alpha_{1}} M^{-\gamma_{1}} S^{-\delta_{1}} Z_{1}+r_{1} k_{1}^{-\beta_{1}} P_{1}^{\alpha_{1} \beta_{1}-1} M^{-\beta_{1} \gamma_{1}} S^{-\beta_{1} \delta_{1}}+P_{1}^{-1} M+P_{1}^{-1} S \leq 1$,

$$
\begin{aligned}
& k_{2} P_{2}^{1-\alpha_{2}} M^{-\gamma_{2}} S^{-\delta_{2}} Z_{2}+r_{2} k_{2}^{-\beta_{2}} P_{2}^{\alpha_{2} \beta_{2}-1} M^{-\beta_{2} \gamma_{2}} S^{-\beta_{2} \delta_{2}}+P_{2}^{-1} M+P_{2}^{-1} S \leq 1, \\
& P_{1}, P_{2}, M, S, Z_{1}, Z_{2}>0 .
\end{aligned}
$$

Model (19) can be solved globally by its dual problem (Duffin et al., 1967).

\section{Numerical example and sensitivity analysis}

Consider the following data

\begin{tabular}{ccccccc}
\hline Market 1 & $k_{1}=10^{6}$ & $r_{1}=4$ & $\alpha_{1}=3$ & $\beta_{1}=0.03$ & $\delta_{1}=0.2$ & $\gamma_{1}=0.4$ \\
\hline Market 2 & $k_{2}=10^{6}$ & $r_{2}=9$ & $\alpha_{2}=2.5$ & $\beta_{2}=0.05$ & $\delta_{2}=0.4$ & $\gamma_{2}=0.3$ \\
\hline
\end{tabular}

For above example, the ideal value of $\Pi_{1}\left(P_{1}, M, S\right)$ and $\Pi_{2}\left(P_{2}, M, S\right)$ are $\Pi_{1}=5967.7$ and $\Pi_{2}=9376.4$. Suppose we want to decide on optimal pricing, marketing and service strategy for a single electronic product in these two competitive markets. The solutions of the proposed model by compromise programming method for $q=1$ and $q=2$ for $w_{1}=w_{2}=0.5$ are given in Table 1 .

\section{Table1}

Optimal values of decision variables for $w_{1}=w_{2}=0.5$

\begin{tabular}{cccccccc}
\hline$q$ & $p_{1}^{*}$ & $p_{2}^{*}$ & $M^{*}$ & $S^{*}$ & $Z_{1}^{*}$ & $Z_{2}^{*}$ & $U_{q}$ \\
\hline 1 & 7.8189 & 13.4219 & 1.1899 & 0.9433 & 5566.2 & 7886.5 & $1.6^{*} 10^{-5}$ \\
2 & 7.5788 & 13.1684 & 1.1318 & 0.8462 & 5675.6 & 7644.9 & $2.5^{*} 10^{-5}$ \\
\hline
\end{tabular}

Figs. 2-3, illustrate the behavior of $Z_{1}^{*}$ and $Z_{2}^{*}$ for different values of $w_{1}$ and $w_{2}$.

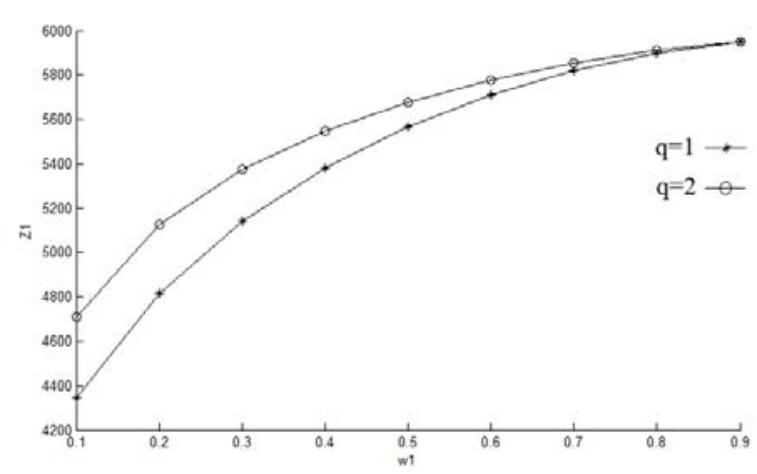

Fig. 1. Behavior of $Z_{1}^{*}$ for different values of $w_{1}$

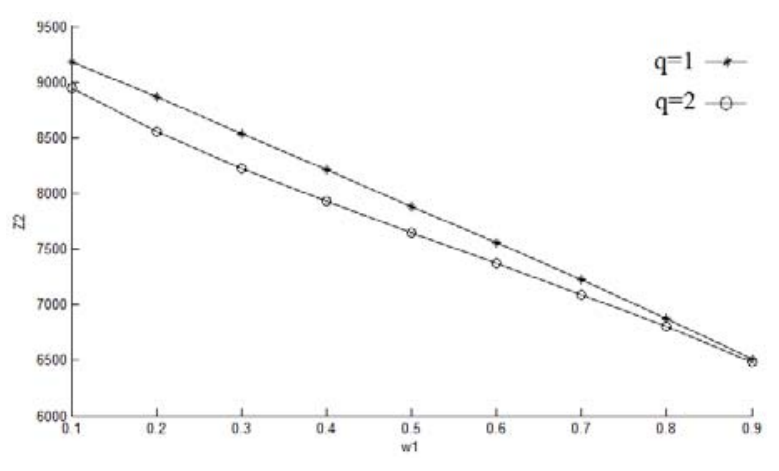

Fig. 2. Behavior of $Z_{2}^{*}$ for different values of $w_{1}$

Fig. (1) and Fig. (2) show that by increasing $w_{1}, Z_{1}^{*}$ increases as a curve and $Z_{2}^{*}$ decreases for $q=1,2$.

\section{Conclusion and future research}

In this paper, we have presented a new multi-objective pricing and marketing planning. The proposed model was solved using compromise programming and the resulted model was transformed into a posynomial form to ensure that global solution is guaranteed. The implementation of the proposed model was studied using some numerical example and the results are discussed in details. As a future research, one can use multiple markets using game strategy. 


\section{Appendix A}

In this section we are interested in finding the value of $\Pi_{1}$. This solution procedure is based on the algorithm explained by Duffin et al. (1967).Consider the following objective function:

$\max \Pi_{1}\left(P_{1}, M, S\right)=k_{1} P_{1}^{1-\alpha_{1}} M^{\gamma_{1}} S^{\delta_{1}}-r_{1} k_{1}^{1-\beta_{1}} P_{1}^{\alpha_{1} \beta_{1}-\alpha_{1}} M^{\gamma_{1}-\beta_{1} \gamma_{1}} S^{\delta_{1}-\beta_{1} \delta_{1}}-k_{1} P_{1}^{-\alpha_{1}} M^{\gamma_{1}+1} S^{\delta_{1}}-k_{1} P_{1}^{-\alpha_{1}} M^{\gamma_{1}} S^{\delta_{1}+1}$, where the model is a signomial GP problem.

$\min T_{1}^{-1}$

subject to $k_{1} P_{1}^{1-\alpha_{1}} M^{\gamma_{1}} S^{\delta_{1}}-r_{1} k_{1}^{1-\beta_{1}} P_{1}^{\alpha_{1} \beta_{1}-\alpha_{1}} M^{\gamma_{1}-\beta_{1} \gamma_{1}} S^{\delta_{1}-\beta_{1} \delta_{1}}-k_{1} P_{1}^{-\alpha_{1}} M^{\gamma_{1}+1} S^{\delta_{1}}-k_{1} P_{1}^{-\alpha_{1}} M^{\gamma_{1}} S^{\delta_{1}+1} \geq T_{1}$, $P_{1}, M, S, T_{1}>0$.

Since, $T_{1}>0$ the first constraint can be transformed into the following form,

$\min T_{1}^{-1}$

subject to $k_{1}^{-1} P_{1}^{\alpha_{1}-1} M^{-\gamma_{1}} S^{-\delta_{1}} T_{1}+r_{1} k_{1}^{-\beta_{1}} P_{1}^{\alpha_{1} \beta_{1}-1} M^{-\beta_{1} \gamma_{1}} S^{-\beta_{1} \delta_{1}}+P_{1}^{-1} M+P_{1}^{-1} S \leq 1$, $P_{1}, M, S, T_{1}>0$.

The above problem is a primal posynomial geometric programming with zero degree of difficulty. The corresponding dual problem is:

$\max \left(\frac{k_{1}^{-1} \lambda}{w_{1}}\right)^{w_{1}}\left(\frac{r_{1} k_{1}^{-\beta_{1}} \lambda}{w_{2}}\right)^{w_{2}}\left(\frac{\lambda}{w_{3}}\right)^{w_{3}}\left(\frac{\lambda}{w_{4}}\right)^{w_{4}}$

subject to $w_{0}=1$,

$$
\begin{aligned}
& -w_{0}+w_{1}=0, \\
& \left(\alpha_{1}-1\right) w_{1}+\left(\alpha_{1} \beta_{1}-1\right) w_{2}-w_{3}-w_{4}=0, \\
& -\gamma_{1} w_{1}-\beta_{1} \gamma_{1} w_{2}+w_{3}=0, \\
& -\delta_{1} w_{1}-\beta_{1} \delta_{1} w_{2}+w_{4}=0, \\
& \lambda=w_{1}+w_{2}+w_{3}+w_{4}, \\
& w_{0}, w_{1}, w_{2}, w_{3}, w_{4}>0 .
\end{aligned}
$$

Therefore we have:

$$
\begin{aligned}
& w_{1}=1, \quad w_{2}=\frac{-\left(\alpha_{1}-\gamma_{1}-\delta_{1}-1\right)}{\left(\alpha_{1} \beta_{1}-\beta_{1} \gamma_{1}-\beta_{1} \delta_{1}-1\right)}, w_{3}=\frac{\left(\beta_{1}-1\right) \gamma_{1}}{\left(\alpha_{1} \beta_{1}-\beta_{1} \gamma_{1}-\beta_{1} \delta_{1}-1\right)}, w_{4}=\frac{\left(\beta_{1}-1\right) \delta_{1}}{\left(\alpha_{1} \beta_{1}-\beta_{1} \gamma_{1}-\beta_{1} \delta_{1}-1\right)} \\
& \lambda=\frac{\alpha_{1}\left(\beta_{1}-1\right)}{\left(\alpha_{1} \beta_{1}-\beta_{1} \gamma_{1}-\beta_{1} \delta_{1}-1\right)} .
\end{aligned}
$$

To have feasible solution for dual problem, we need some additional assumptions to ensure that $w_{1}$ to $w_{4}$ remain positive which are $\alpha_{1}>1+\gamma_{1}+\delta_{1}, \alpha_{1} \beta_{1}-\beta_{1} \gamma_{1}-\beta_{1} \delta_{1}-1<0$. For $i=1, \ldots, 4$ let $\Delta_{i}=\frac{w_{i}}{\lambda}$. Note that $\Delta_{i}$, for $i=1, \ldots, 4$, are the weights of the terms in the constraints of model. In fact $\Delta_{1}$ to $\Delta_{4}$, represent the proportion of revenue $\left(\Delta_{1}\right)$, production cost $\left(\Delta_{2}\right)$, marketing cost $\left(\Delta_{3}\right)$ and service cost. The following relations must hold: 
$\Delta_{1}=k_{1} P_{1}^{1-\alpha_{1}} M^{-\gamma_{1}} S^{-\delta_{1}} T_{1}, \Delta_{2}=r_{1} k_{1}^{-\beta_{1}} P_{1}^{\alpha_{1} \beta_{1}-1} M^{-\beta_{1} \gamma_{1}} S^{-\beta_{1} \delta_{1}}, \Delta_{3}=P_{1}^{-1} M, \Delta_{4}=P_{1}^{-1} S$.

where $\sum_{i=1}^{4} \Delta_{i}=1$ at optimality (Lee \& Kim, 1993). Using above equations, the optimal solution of the problem can be summarized as follow:

$P_{1}=\left(\frac{r}{\Delta_{3}^{\beta_{1} \gamma_{1}} \Delta_{4}^{\beta_{1} \delta_{1}} k^{\beta_{1}} \Delta_{2}}\right)^{\frac{1}{1-\alpha_{1} \beta_{1}+\beta_{1} \gamma_{1}+\beta_{1} \delta_{1}}} \quad S^{*}=\Delta_{4} P^{*} M^{*}=\Delta_{3} P^{*}$

Note that we can find the value of $\Pi_{2}$ by using a similar procedure.

\section{References}

Abad, P. L. (1988). Determining optimal selling price and the lot size when the supplier offers allunit quantity discounts. Decision Sciences, 19, 622-634.

Beightler, C. S., \& Philip, D. T. (1976). Applied Geometric Programming. New York: Wiley.

Bhargava, H. K., Choudhary, V., \& Krishnan, R. (2001). Pricing and Product Design: Intermediary Strategies in an Electronic Market. International Journal of Electronic Commerce, 5 , 37-56.

Chen, Y. A., Wang, H. C., \& Shen, H. Z. (2006). Study on the price competition between ecommerce retailer and conventional retailer. system engineering theory and practice, 26, 35-41.

Chun, S., \& Kim, J. (2005). Pricing strategies in B2C electronic commerce: Analytical and empirical approaches. decision support systems, 40, 375-388.

Duffin, R., Peterson, E. L., \& Zener, C. (1967). Geometric Programming: Theory and Application. New York: Wiley.

Elmaghraby, W., \& Keskinocak, P. (2003). Dynamic pricing in the presence of inventory considerations: research overview, current practices, and future directions. Management Science, 49, 1278-1309.

Fathian, M., Sadjadi, S. J., \& Sajadi, S. (2009). Optimal pricing model for electronic products. computers and industrial engineering, 56, 255-259.

Jornsten, K., \& Uboe, J. (2009). Strategic pricing of commodities. Applied Mathematical Finance, 16 , 385-399.

Jung, H., \& Klein, C. M. (2001). Optimal inventory policies under decreasing cost functions via geometric programming. European Journal of Operational Research, 132, 628-642.

kim, D. S., \& Klein, W. J. (1998). Optimal joint pricing and lot sizing with fixed and variable capacity. European Journal of Operational Research, 109, 212-227.

Lee, K. B., Yu, S., \& Kim, S. J. (2006). Analysis of pricing for e- business companies providing information goods and services. Computers and Industrial Engineering, 51, 72-78.

Lee, W. J. (1993). Determining selling price and order quantity by geometric programming, optimal solution, bounds and sensitivity. Decision Sciences, 24, 76-87.

Lee, W. J., \& Kim, D. S. (1993). Optimal and heuristic decision strategies for integrated production and marketing planning. Decision Sciences, 24, 1203-1213.

Lee, W. J., Kim, D. S., \& Cabot, A. V. (1996). Optimal demand rate, lot sizing, and process reliability improvement decisions. IIE Transactiona, 28, 941-952.

Miettinen, K. M. (1999). Non-linear Multi-objective optimization. Kluwer's academic publisher.

Sadjadi, S. J., Orougee, M., \& Aryanezhad, M. B. (2005). Optimal production and marketing planning. Computational Optimization and Application, 30, 195-203.

Serel, D. (2009). Optimal ordering and pricing in a quick response system. International Journal of Production Economics, 121, 700-714.

Szidorovsky, F., Gershon, M. E., \& Dukstein, L. (1986). Techniques for multi objective decision making in systems managemen. New York: Elsevier. 